\title{
Shaft power measurement for marine propulsion system based on magnetic resonances
}

\author{
Li Qin ${ }^{1,2 a)}$, Xincong Zhou ${ }^{1}$, Yan Gao ${ }^{2}$, Pengju Cao ${ }^{2}$, \\ Jianzhou Quan ${ }^{2}$, and Zhixiong $\mathbf{L i}^{1}$ \\ ${ }^{1}$ School of Energy and Power Engineering, Wuhan University of Technology \\ 130 P.O. Box, 1040 Heping Road, Wuhan 430063, P. R. China \\ ${ }^{2}$ Huangpi Campus, Air Force Radar Academy \\ 288 Huangpu Avenue, Wuhan 430345, P. R. China \\ a)19148644@qq.com
}

\begin{abstract}
Marine shaft power measurement is believed to assess the working condition for marine propulsion system. The traditional equipment uses battery-powered supply but could provide only 2-4 hours continuous monitoring time. To address this issue, a new shaft power measurement technique based on magnetic resonances is proposed in this paper. It was able to transfer $70 \mathrm{~mA}$ over distances in the range of 5 to 20 millimeters. We experimentally demonstrated efficient power transfer over the self-resonant coils in the transmitter to the receiving end in order to achieve the transfer of energy. It is shown that the strain-type measurement using the magnetic resonances technique produces superior results to those using battery-powered measurements, and thus has application importance.
\end{abstract}

Keywords: shaft power, magnetic resonances, marine propulsion system

Classification: Electronic instrumentation and control

\section{References}

[1] A. Kurs, A. Karalis, R. Moffatt, et al., "Wireless Power Transfer via Strongly Coupled Magnetic Resonances," Science, vol. 317, no. 3, pp. 8386, 2007.

[2] V. T. Lamaris and D. T. Hountalas, "A general purpose diagnostic technique for marine diesel engines," Energy Conversion and Management, vol. 51 , no. 4 , pp. $740-753,2010$.

[3] D. Lijun, S. Maosheng, L. Wenli, Z. Gang, and L. Zhigang, "Principle analysis and realization of electric propulsion load simulation system," Transactions of China Electrotechnical Society, vol. 24, no. 7, pp. 70-75, 2009.

[4] A. Grzadziela, "Dynamic analyses of ships shafts lines," Systems Science, vol. 34, no. 3, pp. 45-50, 2008. 


\section{Introduction}

The shaft power is the main performance parameter for the shipyards and the owners of new ships. It can be measured to assess the working condition of the power system, and can determine the technical condition of the matching of propulsion engine to propeller. Marine shafting located between main engine and propeller is composed of transmission shaft, supporting parts, tail-end sealing device and accessories. The main function of the shafting is to transfer the power generated by the marine engine to the propeller, while to transfer the axial force produced by the propeller to the hull to drive the marine navigation.

Phase-type torque sensor takes torsion angle as the measurement object. Flexible shaft will deform under the action of torque, leading to relative rotation of any two of its cross-section occurs. So a phase angle is generated. The torque that shaft suffered can be obtained by measuring the phase angle. Generally the phase angle of shaft can be measured through steel wire sensors, strain gauge, and laser measuring method, etc. Until the 1980s and 1990s, the steel wire sensor is still used in the measurement of marine diesel engine shaft power. The system accuracy is high, but different shaft sizes require the appropriate matching collector ring. In addition, this approach is very costly, and a portion of the equipments is very cumbersome to be installed in the shaft, especially for the large marines or ships with small installation rooms, some equipments can not incorporated into the measurement system.

The strain gauge torque instrument measures the main strain under the action of torque through the strain sensor, such as strain gauge, ferromagnetic materials. The resistance strain gauges are commonly used in marine shaft. There will be stress on the surface when the shaft rotates and it will transfer to the strain gauge attached to the test shaft on the surface. The resistance of strain gauge changes along with the change of stress. The change in strain gauge resistance is proportional to the change of the strain on the surface of the shaft. By measuring the resistance of strain gauges, the shaft power can be calculated.

\section{Signal transmission mode of the test systems}

As an important method of measuring the conversion efficiency, the measuring of shaft power has become a major parameter in new ships' inspection and acceptance for shipyards and ship owners. The intention of the shaft power measurement is to enable the ship's crew and, consequently, the ship owner's operation management to optimize the operation parameters of ships, and to improve the power, economy and security. Currently, the signal transmission mode of shaft power test systems mainly includes contact-type torque measurement and telemetry torque measurement.

The principle of contact-type torque measurement is that the signal transmitted through the contacts of collector ring and brush generated by the torque meter, then it reaches to the computer through the data acquisition device, and shaft power value can be got after data processing. However, due 
to the existence of contact resistance between the collector rings and brushes, it produces a lot of interference on the measurement results, so it cannot be used for vibration and high-speed measurement.

The telemetry torque measurement collects the shaft torque signal by the sending and receiving of wireless signals. When installing, the signal transmitter is fixed on the shaft with the axis rotation, the torque signal generated by the wire is connected to the transmitter, the torque signal is output from the transmitter to the receiving device, and then transfer the shaft power to the computer for data analysis and processing.

There is sliding ring type or battery type for the power supply system of the transmitter which is fixed to the rotating shaft. The slip-ring and carbon-brush power supply system is bulky and is used for shaft low-speed rotation, generally is less than $500 \mathrm{r} / \mathrm{min}$. Battery-powered type due to the simple structure, small size, is widely used. But there is only 2-4 hours of continuous power supply time for battery type. For dynamic monitoring of ship shafting, it is difficult to replace the battery to stop the operation of the shaft.

\section{Wireless power transmissions}

Due to the motion characteristics of the shaft, the traditional strain-type shaft power measurement equipment uses battery-powered wireless power supply. As the limited capacity of the battery, the battery service life is generally two hours in the ship shafting shaft power test. Therefore, frequent replacement of the battery or frequent recharging is needed. The use of the equipment is inconvenient. The problems of lack of electricity cannot be timely supply.

Wireless power transmission is passed by means of the electromagnetic field or electromagnetic wave to transfer energy. June 7, 2007 MIT Assistant Professor Marin Soljacic realized the wireless power supply of the bulb using the magnetically coupled resonance between matched antennas, which is called WiTricity.

In the experiment, two diameter $50 \mathrm{~cm}$ copper coils are used as a resonator. The transmitter vibrates at $10 \mathrm{MHz}$ by adjusting the transmission frequency. It is not the ordinary electromagnetic waves, but is the nonradiative magnetic field formed in the surroundings. It converts electrical energy to the magnetic field between the two coils form an invisible energy channels. A $60 \mathrm{~W}$ bulb in the distance of 2 meters is lit successfully, which makes it possible to realize the wireless transmission of electrical energy. The core of the method is magnetically coupled resonance.

At present, the magnetic coupling is used for short distances in order to recharge the battery. It requires that the device you are charging is as close to the induction coil as possible, this is because the magnetic energy is lost rapidly with the distance. In the conventional magnetic induction, distance can only be increased by increasing the magnetic field strength. On the other hand, in the help of WiTricity, the magnetic coupling occurred within 
a few feet away, without the need to enhance the magnetic field strength. This technology has made a definite breakthrough in the charging of electric vehicles, small robots and portable mobile devices.

Magnetic resonance wireless power supply circuit breaks through the traditional wired power supply mode, achieves a safe and effective mobile power supply.

\section{Application in the measurement of shaft power}

Large marine shaft power measurement often adopts telemetry strain method, the advantages are lightweight, easy installation, environmental adaptability, and with high accuracy and reliability, it can be used in full-scale ship shaft power measurement. With the rapid development of shipbuilding industry, the speed and powering, high efficiency and energy conservation have become important indicators of shipbuilding. While the shaft power measurement is an important means for the calculation of the conversion efficiency. With the measurement of shaft power in different operation conditions, the matching of propulsion engine to propeller can be inspected, and it is an important basis for the matching of propulsion engine to propeller of old ships.

The wireless sensor power supply of marine is composed of the fixed end wireless transmitter module, rotation demodulation receiver module and antenna attachments. The wireless power supply circuit includes both transmitter and receiver as shown in Fig. 1.

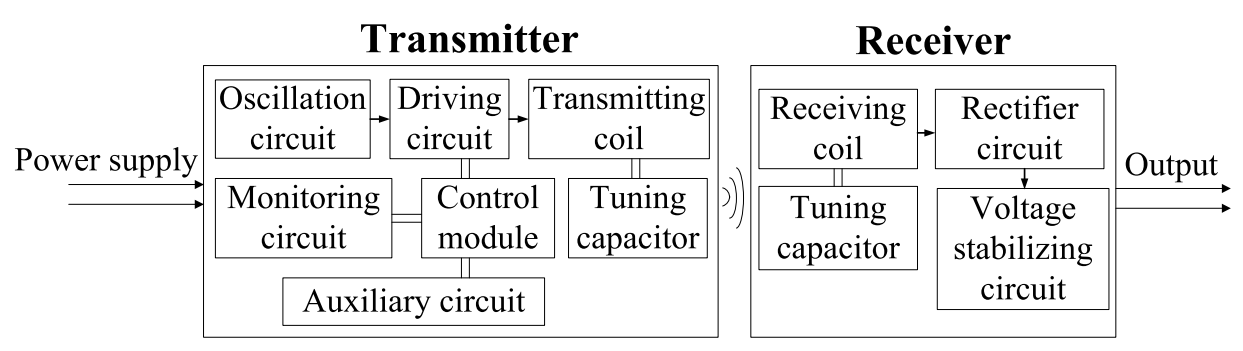

Fig. 1. Wireless power supply circuit block diagram.

The transmitter is consist of the oscillation circuit, the driving circuit, the auxiliary circuit, the control module, the monitoring circuit, the tuning capacitor and the sending coil. The receiver includes the receiving coil, the rectifier circuit, the voltage stabilizing circuit and the tuning capacitor.

Marine propulsion shafting system is a multi-support, easy deformation dynamic system with large-scale and high-mass. The conventional method used for the measurement of power transferred by rotating shafts is the use of strain gauges glued to the shaft and connected to signal conditioning amplifiers mounted on the shaft.

The experiment based on magnetic resonances was applied on a certain marine propulsion shafting system as shown in Fig. 2. Strain gauges were fixed in the tail of the intermediate shaft bearing about $1.8 \mathrm{~m}-2.0 \mathrm{~m}$. The type of the measured marine host is B\&W 6S70MC, rated power is $13364 \mathrm{~kW}$, and 
the minimum speed of the shaft is $44 \mathrm{rpm}$, the maximum speed is to $65 \mathrm{rpm}$. The shafting system consists of first shaft, intermediate shaft, stern shaft and bearings. The intermediate shaft diameter is $500 \mathrm{~mm}$. The whole shaft is hollow with diameter of $25 \mathrm{~mm}$. The marine shafting system test results are shown in the fitting curve of the power with speed in Fig. 3.

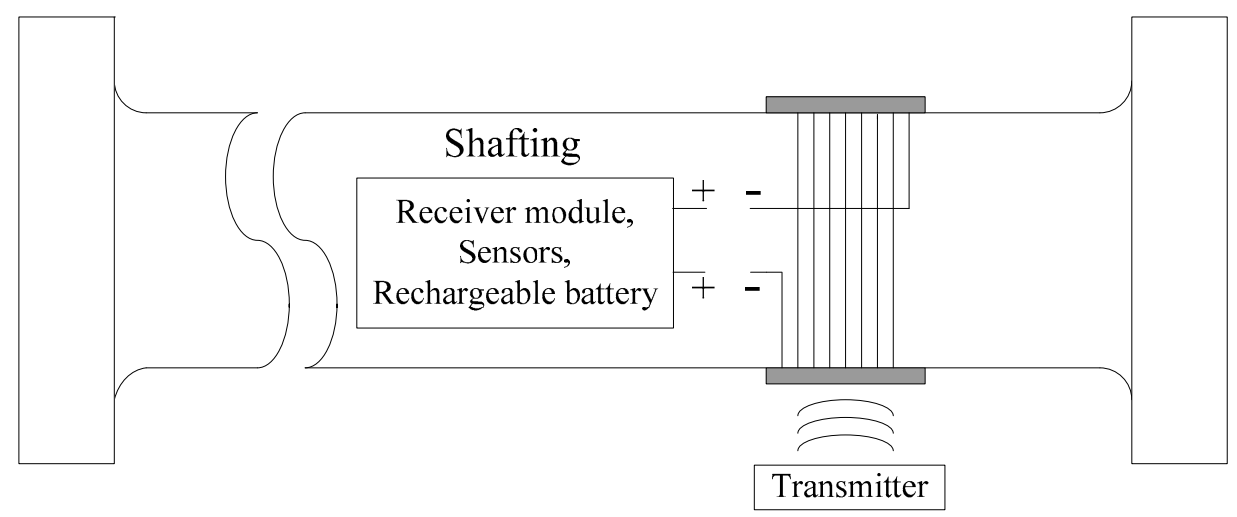

Fig. 2. Power supply device in the marine shafting performance measuring equipment.



Fig. 3. Fitting curve of the power with speed.

The shaft power dynamic monitoring experiment lasted for hundreds of hours, the average shaft power with the change of speed was measured. It can be seen from the fitted curve that the change of power of the host along with the speed gone smoothly, approximately $9540 \mathrm{~kW}$ of shaft power could be got at the highest rated speed $65 \mathrm{r} / \mathrm{min}$, and the maximum shaft power of host was up to $15835 \mathrm{~kW}$, which is consistent with the rated power value. The test results meet the design requirements.

\section{Conclusions}

The normal working of marine is determined by the reliable operation of marine shafting. As the shaft power measurement is carried out in the dynamic 
circumstance, the measurement requires high stability and dynamic monitoring. Due to the limitations of battery-powered shaft power measurement system, conventional equipment could not monitor the dynamic information of shaft power. For this reason, a new equipment based on magnetic resonances was presented to monitor the changing tendency of power on line in this paper. We experimentally demonstrated efficient power transfer over the self-resonant coils in the transmitter to the receiving end. The electromagnetic wave frequency of the transmitter was consistent with the receiving end of the resonant frequency, resonance was produced and efficient power was transferred. The experimental test results have shown that the presented equipment is effective for the dynamic monitoring of shaft power. Further research will extend the proposed monitoring system to different types of ships.

\section{Acknowledgments}

This project is sponsored by the grants from the National Natural Sciences Foundation of China (No. 51139005, No. 51079119 and No. 50979084), and the Fundamental Research Funds for the Central Universities (No. 2012-JL$01)$. 